\title{
SPECTRAL ENCLOSURES FOR NON-SELF-ADJOINT DISCRETE SCHRÖDINGER OPERATORS
}

\author{
ORIF O. IBROGIMOV AND FRANTIŠEK ŠTAMPACH
}

\begin{abstract}
We study location of eigenvalues of one-dimensional discrete Schrödinger operators with complex $\ell^{p}$-potentials for $1 \leq p \leq \infty$. In the case of $\ell^{1}$-potentials, the derived bound is shown to be optimal. For $p>1$, two different spectral bounds are obtained. The method relies on the Birman-Schwinger principle and various techniques for estimations of the norm of the Birman-Schwinger operator.
\end{abstract}

\section{INTRODUCTION AND MAIN RESULTS}

Recent years have seen a significant development in the spectral theory of non-selfadjoint operators. A great deal of research aims to a localization of spectra of differential operators such as Schrödinger operators [1, 3, 9, 12, 16, 18, 23, 25, 29, 31, 33, Dirac operators 6, 10, 17] and others [8, 24, 27. For related recent results obtained in an abstract operator theoretic setting, the reader may also consult $2,11,13,14$.

In contrast to the differential operators, there are almost no works studying similar questions for their discrete analogues, i.e. the difference operators like discrete Schrödinger or discrete Dirac operators with complex potentials. The authors are only aware of [26] which is focused on the number rather than the location of eigenvalues for the discrete Schrödinger operator with a complex potential and a related work [28] for the cubic lattice. Some constrains on the location of the discrete spectrum of semi-infinite complex Jacobi matrices are discussed in [15].

Our main interest is to bridge this gap focusing first on the location of spectrum of the one-dimensional dicrete Schrödinger operator with a complex valued $\ell^{p}$-potential, $1 \leq p \leq \infty$, which is the objective of this paper. The one-dimensional Dirac operator with a complex potential is discussed in a separate paper 4 .

Let $H_{0}$ be the discrete Laplacian acting on the Hilbert space $\ell^{2}(\mathbb{Z})$ which is the bounded operator determined by the equation

$$
H_{0} e_{n}:=e_{n-1}+e_{n+1}, \quad \forall n \in \mathbb{Z},
$$

where $\left\{e_{n}\right\}_{n \in \mathbb{Z}}$ stands for the standard basis of $\ell^{2}(\mathbb{Z})$. Further, to a given complex sequence $v=\left\{v_{n}\right\}_{n \in \mathbb{Z}}$, we define the potential $V$ as a diagonal operator

$$
V e_{n}:=v_{n} e_{n}, \quad \forall n \in \mathbb{Z}
$$

Date: October 28, 2019.

2010 Mathematics Subject Classification. 34L15, 47B36, 47A75.

Key words and phrases. Discrete Schrödinger operator, Birman-Schwinger principle point spectrum, Jacobi matrix. 
The matrix representation of the discrete Schrödinger operator $H_{V}:=H_{0}+V$ is the doubly-infinite Jacobi matrix

$$
H_{V}=\left(\begin{array}{ccccccc}
\ddots & \ddots & \ddots & & & & \\
& 1 & v_{-1} & 1 & & & \\
& & 1 & v_{0} & 1 & & \\
& & & 1 & v_{1} & 1 & \\
& & & & \ddots & \ddots & \ddots
\end{array}\right) .
$$

It is well known that the spectrum of the unperturbed operator $H_{0}$ is absolutely continuous and covers the interval $[-2,2]$. If the potential $V$ vanishes at infinity, i.e. $v_{n} \rightarrow 0$ as $n \rightarrow \pm \infty$, then $V$ is compact and the essential spectrum of the perturbed operator $H_{V}=H_{0}+V$ coincides with the interval $[-2,2]$.

The goal of the present paper is to investigate the location of the spectrum of $H_{V}$ with an $\ell^{p}$-potential $V$ for $1 \leq p \leq \infty$. Our primary result concerns the location of eigenvalues of $H_{V}$ with an $\ell^{1}$-potential. Moreover, the obtained bound is shown to be optimal; see Theorem 1 . If the decay of the potential is slower, namely if $v \in \ell^{p}(\mathbb{Z})$ for $1<p \leq \infty$, we derive bounds for the entire spectrum of $H_{V}$; see Theorems 2 and 3 .

Theorem 1. Let $v \in \ell^{1}(\mathbb{Z})$. Then

$$
\sigma_{\mathrm{p}}\left(H_{V}\right) \subset\left\{\lambda \in \mathbb{C} \backslash(-2,2)|| \lambda^{2}-4 \mid \leq\|v\|_{\ell^{1}(\mathbb{Z})}^{2}\right\} .
$$

The bound in (1.1) is sharp in the following sense: For any $Q>0$ and any point $\lambda \in$ $\mathbb{C} \backslash(-2,2)$ which fulfills the equation

$$
\left|\lambda^{2}-4\right|=Q^{2}
$$

there exists $v \in \ell^{1}(\mathbb{Z})$ such that $Q=\|v\|_{\ell^{1}(\mathbb{Z})}$ and $\lambda$ is an eigenvalue of the corresponding discrete Schrödinger operator $H_{V}$, see Section 4 . This means that every boundary point of the spectral enclosure (1.1), with the exception of points located in $(-2,2)$, is an eigenvalue of $H_{V}$ for some $\ell^{1}$-potential $V$. Hence the obtained spectral bound cannot be squeezed any further.

Clearly, the geometry of the spectral enclosure 1.1 depends on the $\ell^{1}$-norm of the potential. If $\|v\|_{\ell^{1}(\mathbb{Z})} \leq 2$, the spectral enclosure consists of two connected components each containing either the point 2 or -2 . If $\|v\|_{\ell^{1}(\mathbb{Z})}>2$, the spectral enclosure is a connected set containing the entire interval $[-2,2]$. The set is always symmetric with respect to both the real and the imaginary axes, see Figure 1 in Section 5.

Our next result provides a spectral estimate in terms of the $\ell^{p}$-norm of the potential. In the sequel, for $p \in(1, \infty]$, we denote by $q \in[1, \infty)$ the corresponding Hölder exponent, i.e. $q=p /(p-1)$ if $1<p<\infty$ and $q=1$ if $p=\infty$.

Theorem 2. Let $1<p \leq \infty$ and let $v \in \ell^{p}(\mathbb{Z})$. Then

$$
\sigma\left(H_{V}\right) \subset\left\{k+\frac{1}{k}|k \in \mathbb{C} \backslash\{0\},| k \mid \leq 1 \text { and }\left|k-\frac{1}{k}\right|\left(\frac{1-|k|^{q}}{1+|k|^{q}}\right)^{1 / q} \leq\|v\|_{\ell^{p}(\mathbb{Z})}\right\} .
$$

Similarly to the continuous setting, see e.g. [7, 21, complex interpolation applied to an appropriate analytic family of Birman-Schwinger type operators yields the following alternative spectral enclosure for the case of $\ell^{p}$-potentials.

Theorem 3. Let $1<p \leq \infty$ and let $v \in \ell^{p}(\mathbb{Z})$. Then

$$
\sigma\left(H_{V}\right) \subset\left\{\lambda \in \mathbb{C}|| \lambda^{2}-4 \mid \operatorname{dist}(\lambda,[-2,2])^{2 p-2} \leq\|v\|_{\ell^{p}(\mathbb{Z})}^{2 p}\right\} .
$$

Remark 1. For $p=\infty$, the inequality in 1.3 has to be understood as

$$
\operatorname{dist}(\lambda,[-2,2]) \leq\|v\|_{\ell^{\infty}(\mathbb{Z})} .
$$


Note also that, if one sets $p=1$ in $(1.3)$, one arrives at the bound $(1.1)$ without the exclusion of the interval $(-2,2)$, however.

Notice the comparatively simpler form of the spectral enclosure from Theorem 1 in comparison with the ones of Theorems 2 and 3 . The spectral enclosures from 1.2 and 1.3 are compact sets which are symmetric with respect to both the real and the imaginary axes. However, in contrast to (1.1), they are always connected sets containing the entire interval $[-2,2]$. In general, none of the spectral enclosures from $(1.2)$ and (1.3) is a subset of the other. Naturally, taking their intersection yields the best result. Several plots of boundary curves of these sets as well as their comparison are presented in Section 5 .

The methodology used to deduce spectral enclosures of Theorems 1 and 2 is by no means new. It relies on the conventional Birman-Schwinger principle similarly as the analogous results for the (continuous) Schrödinger and Dirac operators, see e.g. 10, 17 , 19, 20,23 and also 11,27. The lastly mentioned papers served as a motivation for the current work. In comparison with the (continuous) Schrödinger operators, where the spectral enclosure is a disk centered at the origin, the spectral enclosures from Theorems 1, 2 and 3 have more interesting geometry. On the other hand, to estimate the norm of the Birman-Schwinger operator is technically less demanding in the discrete setting. Namely, it is done by elementary means in the case of $\ell^{1}$-potentials, it makes use of either the Schur test or discrete Young's inequality to deduce $\sqrt{1.2}$, or it employs a very particular form of Stein's complex interpolation to obtain (1.3).

The outline of the paper is as follows. In Section 2, the Birman-Schwinger principle is briefly recalled. Section 3 contains proofs of Theorems 1, 2, and 3 with two alternative proofs for Theorem 2, In Section 4, we discuss an example of the operator $H_{V}$ with a delta potential which demonstrates the optimality of the spectral enclosure (1.1) for $\ell^{1}$-potentials. Final Section 5 is devoted to numerical illustrations of the results of Theorems 1, 2, and 3 .

\section{The Birman-Schwinger Principle}

The central role in our analysis is played by the Birman-Schwinger operator

$$
K(\lambda):=|V|^{1 / 2}\left(H_{0}-\lambda\right)^{-1} V_{1 / 2}
$$

where $|V|^{1 / 2} e_{n}=\sqrt{\left|v_{n}\right|} e_{n}$ and $V_{1 / 2} e_{n}=\operatorname{sgn}\left(v_{n}\right) \sqrt{\left|v_{n}\right|} e_{n}$ with the complex signum function sgn: $\mathbb{C} \rightarrow \mathbb{C}$ defined by

$$
\operatorname{sgn} z=\left\{\begin{array}{cc}
\frac{z}{|z|} & \text { if } z \neq 0, \\
0 & \text { if } z=0 .
\end{array}\right.
$$

For $\lambda \in \mathbb{C} \backslash[-2,2]$, the Birman-Schwinger operator is a bounded operator in $\ell^{2}(\mathbb{Z})$ and its matrix elements read

$$
K(\lambda)_{m, n}=\sqrt{\left|v_{m}\right|}\left(H_{0}-\lambda\right)_{m, n}^{-1} \sqrt{\left|v_{n}\right|} \operatorname{sgn}\left(v_{n}\right), \quad \forall m, n \in \mathbb{Z} .
$$

Here the middle term corresponds to the matrix element of the resolvent of the discrete Laplace operator $H_{0}$ which is given by

$$
\left(H_{0}-\lambda\right)_{m, n}^{-1}=\left(H_{0}-k-k^{-1}\right)_{m, n}^{-1}=\frac{k^{|m-n|}}{k-k^{-1}}, \quad \forall m, n \in \mathbb{Z},
$$

where $0<|k|<1$ and $\lambda=k+k^{-1}$, see [36. Chp. 1]. Here, it is often convenient to relate the spectral parameter $\lambda$ with $k$ by the Joukowsky transform $\lambda=k+k^{-1}$ which is a bijection between the punctured unit disk $0<|k|<1$ and the resolvent set $\mathbb{C} \backslash[-2,2]$ of $H_{0}$. 
For $\lambda \in \mathbb{C} \backslash[-2,2]$ and a bounded potential $V$, the Birman-Schwinger principle is the equivalence

$$
\lambda \in \sigma\left(H_{V}\right) \quad \Longleftrightarrow \quad-1 \in \sigma(K(\lambda))
$$

can be easily justified by usual arguments. In particular, it follows that the implication

$$
\|K(\lambda)\|<1 \quad \Longrightarrow \quad \lambda \notin \sigma\left(H_{V}\right)
$$

holds true for $\lambda \notin[-2,2]$. Moreover, the implication $\Longrightarrow$ in 2.3 remains valid even if the spectra are replaced by point spectra. Indeed, if $H_{V} \psi=\lambda \psi$ for some $\lambda \in \mathbb{C} \backslash[-2,2]$ and $\psi \in \ell^{2}(\mathbb{Z})$, then simple manipulations with the eigenvalue equation yields $K(\lambda) \phi=-\phi$ for $\phi:=|V|^{1 / 2} \psi \in \ell^{2}(\mathbb{Z})$ since $|V|^{1 / 2}$ is bounded by our assumption. Consequently, the Birman-Schwinger principle implies the inclusion

$$
\sigma_{p}\left(H_{V}\right) \subset\{\lambda \in \mathbb{C} \backslash[-2,2] \mid\|K(\lambda)\| \geq 1\} \cup[-2,2] .
$$

\section{ProOFs}

3.1. Proof of Theorem 1. The proof uses 2.5). Since the set on the right-hand side of (1.1) always contains points \pm 2 we can distinguish the following two cases.

Case $\lambda \notin[-2,2]$. Let $k \in \mathbb{C}$ be such that $0<|k|<1$ and $\lambda=k+k^{-1}$. It follows readily from 2.2 that

$$
\left|\left(H_{0}-\lambda\right)_{m, n}^{-1}\right|<\frac{1}{\left|k-k^{-1}\right|}=\frac{1}{\sqrt{\left|\lambda^{2}-4\right|}}, \quad \forall m, n \in \mathbb{Z} .
$$

For $v \in \ell^{1}(\mathbb{Z})$, the Birman-Schwinger operator $K(\lambda)$ is Hilbert-Schmidt (even trace class). Using 3.1 and the Cauchy-Schwarz inequality, we can estimate

$$
\begin{aligned}
\|K(\lambda) \psi\|_{\ell^{2}(\mathbb{Z})}^{2} & \leq \sum_{m \in \mathbb{Z}}\left(\sum_{n \in \mathbb{Z}} \sqrt{\left|v_{m}\right|}\left|\left(H_{0}-\lambda\right)_{m, n}^{-1}\right| \sqrt{\left|v_{n}\right|}\left|\psi_{n}\right|\right)^{2} \\
& \leq \frac{\|v\|_{\ell^{1}(\mathbb{Z})}}{\left|\lambda^{2}-4\right|}\left(\sum_{m \in \mathbb{Z}} \sqrt{\left|v_{n}\right|}\left|\psi_{n}\right|\right)^{2} \leq \frac{\|v\|_{\ell^{1}(\mathbb{Z})}^{2}}{\left|\lambda^{2}-4\right|}\|\psi\|_{\ell^{2}(\mathbb{Z})}^{2},
\end{aligned}
$$

for any $\psi \in \ell^{2}(\mathbb{Z})$. Hence

$$
\|K(\lambda)\| \leq \frac{\|v\|_{\ell^{1}(\mathbb{Z})}}{\sqrt{\left|\lambda^{2}-4\right|}}
$$

and, according to 2.5), if $\lambda \in \sigma_{p}\left(H_{V}\right)$, then

$$
\left|\lambda^{2}-4\right| \leq\|v\|_{\ell^{1}(\mathbb{Z})}^{2}
$$

Case $\lambda \in(-2,2)$. We make use of the fact that assumption $v \in \ell^{1}(\mathbb{Z})$ actually implies $(-2,2) \cap \sigma_{p}\left(H_{V}\right)=\emptyset$. This seems to be a commonly known result but we did not find an exact reference for this claim concerning doubly-infinite Jacobi matrices with complex entries. Nevertheless, it follows readily from the existence of a particular solution $\phi(k)=$ $\phi_{n}(k)$ of the three-term recurrence

$$
\phi_{n+1}+\left(v_{n}-k-k^{-1}\right) \phi_{n}+\phi_{n-1}=0
$$

which satisfies

$$
\lim _{n \rightarrow \infty} k^{-n} \phi_{n}(k)=1, \quad \forall k \in \mathbb{C},|k| \leq 1, k \neq \pm 1,
$$

provided that $v \in \ell^{1}(\mathbb{Z})$. The solution $\phi(k)$ is referred to as the Jost solution and its existence is proved, for example, in [34, Sec. 13.6] for real valued $v$. However, the reality of the potential is of no importance for the proof and hence the statement can be extended to complex valued $v$ as well. 
If $\lambda \in(-2,2)$ and $k+k^{-1}=\lambda$, then $|k|=1$ but $k \neq \pm 1$. Consequently, the Jost solution $\phi(k)$ exists. Moreover, the second linearly independent solution of (3.4) can be chosen as $\phi(\bar{k})$ since the Wronskian

$$
W(\phi(k), \phi(\bar{k}))=\lim _{n \rightarrow \infty}\left(\phi_{n}(k) \phi_{n+1}(\bar{k})-\phi_{n+1}(k) \phi_{n}(\bar{k})\right)=2 \mathrm{i} \Im k \neq 0,
$$

where we have used (3.5) and the fact that $\bar{k}=k^{-1}$. Consequently, any solution $\psi$ of the eigenvalue equation $H_{V} \psi=\lambda \psi$, for $\lambda \in(-2,2)$, is a linear combination of $\phi(k)$ and $\phi(\bar{k})$ where $\lambda=k+k^{-1}$ and $|k|=1$. Such $\psi$ is square summable only if trivial. Thus $\lambda$ cannot be an eigenvalue of $H_{V}$. This completes the proof.

3.2. Proof of Theorem 2, Let $k \in \mathbb{C}$ be such that $0<|k|<1$ and $\lambda=k+k^{-1}$. It follows readily from 2.2 that

$$
\left|\left(H_{0}-\lambda\right)_{m, n}^{-1}\right| \leq \frac{|k|^{|m-n|}}{\left|k-k^{-1}\right|}, \quad \forall m, n \in \mathbb{Z}
$$

There are at least two proofs of Theorem 2. The first one makes use of a slight modification of the classical Schur test adjusted to our needs. The classical Schur test can be found in [5, Exer. 9, Chp. II].

Lemma 1 (Schur test). Let $K$ be a bounded operator on $\ell^{2}(\mathbb{Z})$ with matrix entries with respect to the standard basis denoted by $K_{m, n}$ for $m, n \in \mathbb{Z}$. Suppose that there is a set $\mathcal{Z} \subset \mathbb{Z}$ such that $K_{m, n}=0$ whenever $m \in \mathcal{Z}$ or $n \in \mathcal{Z}$. Then, for arbitrary weights $p_{j}>0, j \in \mathcal{I}:=\mathbb{Z} \backslash \mathcal{Z}$, one has

$$
\|K\| \leq\left(\sup _{m \in I} \frac{1}{p_{m}} \sum_{n \in \mathcal{I}} p_{n}\left|K_{m, n}\right|\right)^{1 / 2}\left(\sup _{n \in I} \frac{1}{p_{n}} \sum_{m \in \mathcal{I}} p_{m}\left|K_{m, n}\right|\right)^{1 / 2} .
$$

The second proof applies discrete Young's inequality which can be proved by a simple modification of the proof of classical Young's inequality [32, Thm. 4.2].

Theorem 4 (discrete Young's inequality). Let $p, q, r \geq 1$ be such that

$$
\frac{1}{p}+\frac{1}{q}+\frac{1}{r}=2
$$

Then, for any $f \in \ell^{p}(\mathbb{Z}), g \in \ell^{q}(\mathbb{Z})$, and $h \in \ell^{r}(\mathbb{Z})$, one has

$$
\sum_{i, j \in \mathbb{Z}}\left|f_{i}\left\|g_{j-i}\right\| h_{j}\right| \leq\|f\|_{p}\|g\|_{q}\|h\|_{r}
$$

The first proof of Theorem 2. We apply the Schur test to the Birman-Schwinger operator $K(\lambda)$ defined in 2.1]. Since $K_{m, n}(\lambda)=0$ whenever $v_{n}=0$ or $v_{n}=0$, we put $\mathcal{I}:=\left\{j \in \mathbb{Z} \mid v_{j} \neq 0\right\}$ and the positive weights are chosen as $p_{j}:=\sqrt{\left|v_{j}\right|}$ for $j \in \mathcal{I}$. Then, taking the definition 2.1) and 3.6 into account, we obtain the estimate

$$
\|K(\lambda)\| \leq \frac{1}{\left|k-k^{-1}\right|} \sup _{m \in \mathcal{I}} \sum_{n \in \mathbb{Z}}\left|v_{n}\right||k|^{|m-n|} .
$$

For the case $p=\infty$, we infer from 3.8 that

$$
\|K(\lambda)\| \leq \frac{\|v\|_{\ell^{\infty}(\mathbb{Z})}}{\left|k-k^{-1}\right|} \sum_{n \in \mathbb{Z}}|k|^{|n|}=\frac{\|v\|_{\ell^{\infty}(\mathbb{Z})}}{\left|k-k^{-1}\right|} \frac{1+|k|}{1-|k|} .
$$

Now consider the case $p \in(1, \infty)$. For every fixed $m \in I$, Hölder's inequality yields

$$
\sum_{n \in \mathbb{Z}}\left|v_{n}\right||k|^{|m-n|} \leq\|v\|_{\ell^{p}(\mathbb{Z})}\left(\sum_{n \in \mathbb{Z}}|k|^{|n| q}\right)^{1 / q}=\|v\|_{\ell^{p}(\mathbb{Z})}\left(\frac{1+|k|^{q}}{1-|k|^{q}}\right)^{1 / q}
$$


and thus

$$
\|K(\lambda)\| \leq \frac{\|v\|_{\ell^{p}(\mathbb{Z})}}{\left|k-k^{-1}\right|}\left(\frac{1+|k|^{q}}{1-|k|^{q}}\right)^{1 / q} .
$$

In view of the estimates $(3.9)$ and $(3.11)$, the Birman-Schwinger principle (2.3) thus implies that $\lambda=k+k^{-1} \in \mathbb{C} \backslash[-2,2]$ cannot belong to the spectrum of $H_{V}$ unless it holds that

$$
\|v\|_{\ell^{p}(\mathbb{Z})} \geq\left|k-\frac{1}{k}\right|\left(\frac{1-|k|^{q}}{1+|k|^{q}}\right)^{1 / q}
$$

Finally, noticing that also the interval $[-2,2]$ is always included in the set on the righthand side of $(1.2)$, corresponding to the case when $|k|=1$, the proof is completed.

The second proof of Theorem 2, Suppose $v \in \ell^{p}(\mathbb{Z})$ for $1<p \leq \infty$ and $\lambda \in \mathbb{C} \backslash[-2,2]$ and $k \in \mathbb{C}, 0<|k|<1$, such that $\lambda=k+k^{-1}$. For $\phi, \psi \in \ell^{2}(\mathbb{Z})$, we have

$$
|\langle\phi, K(\lambda) \psi\rangle| \leq \frac{1}{\left|k-k^{-1}\right|} \sum_{m, n \in \mathbb{Z}}\left|f_{m}\right|\left|g_{n-m}\right|\left|h_{m}\right|,
$$

where

$$
f_{n}:=\sqrt{\left|v_{n}\right|} \phi_{n}, \quad g_{n}:=|k|^{|n|}, \quad h_{n}:=\sqrt{\left|v_{n}\right|} \psi_{n}, \quad n \in \mathbb{Z} .
$$

Notice that, by Hölder's inequality, we have

$$
\|f\|_{\ell^{2 p /(p+1)}(\mathbb{Z})} \leq \sqrt{\|v\|_{\ell^{p}(\mathbb{Z})}}\|\phi\|_{\ell^{2}(\mathbb{Z})} \quad \text { and } \quad\|h\|_{\ell^{2 p /(p+1)}(\mathbb{Z})} \leq \sqrt{\|v\|_{\ell^{p}(\mathbb{Z})}}\|\psi\|_{\ell^{2}(\mathbb{Z})} .
$$

Moreover, for any $q \geq 1$, it is clearly true that

$$
\|g\|_{\ell^{q}(\mathbb{Z})}=1+2\left(\sum_{n=1}^{\infty}|k|^{q n}\right)^{1 / q}=\left(\frac{1+|k|^{q}}{1-|k|^{q}}\right)^{1 / q} .
$$

Thus, we can apply Young's inequality (3.7) in (3.13) with the indices $p$ and $r$ replaced by $2 p /(p+1)$ (or by 2 if $p=\infty)$ and $q$ is the Hölder dual index to $p(q=1$ if $p=\infty$ ). Taking also 3.14 and (3.15) into account, we obtain the estimate

$$
|\langle\phi, K(\lambda) \psi\rangle| \leq \frac{\|v\|_{\ell^{p}(\mathbb{Z})}}{\left|k-k^{-1}\right|}\left(\frac{1+|k|^{q}}{1-|k|^{q}}\right)^{1 / q}\|\phi\|_{\ell^{2}(\mathbb{Z})}\|\psi\|_{\ell^{2}(\mathbb{Z})},
$$

for all $\phi, \psi \in \ell^{2}(\mathbb{Z})$. In other words, we arrived at 3.11$)$ again and the same reasoning as in the previous proof implies the desired inclusion (2.4).

3.3. Proof of Theorem 3. The proof makes use of Stein's complex interpolation theorem, see [35. Thm. 1]. However, only a special version of the theorem is sufficient for our needs. Its statement is as follows.

Theorem 5 (Stein's interpolation). Let $T_{z}: \ell^{2}(\mathbb{Z}) \rightarrow \ell^{2}(\mathbb{Z})$ be a family of operators analytic in the strip $0<\Re z<1$ and continuous and uniformly bounded in its closure $0 \leq \Re z \leq 1$. Suppose further that there exist constants $A_{0}$ and $A_{1}$ such that

$$
\left\|T_{\mathrm{i} y}\right\| \leq A_{0} \quad \text { and } \quad\left\|T_{1+\mathrm{i} y}\right\| \leq A_{1},
$$

for all $y \in \mathbb{R}$. Then, for any $\theta \in[0,1]$, one has

$$
\left\|T_{\theta}\right\| \leq A_{0}^{1-\theta} A_{1}^{\theta} \text {. }
$$

Proof of Theorem 3. Let $\lambda \in \mathbb{C} \backslash[-2,2]$ be fixed. If $p=\infty$, one readily estimates the Birman-Schwinger operator getting

$$
\|K(\lambda)\| \leq\left\||V|^{1 / 2} \mid\right\|\left\|\left(H_{0}-\lambda\right)^{-1}\right\|\left\|V_{1 / 2}\right\| \leq \frac{\|v\|_{\infty}}{\operatorname{dist}(\lambda,[-2,2])} .
$$

Then 2.4 implies 1.3 in the particular case $p=\infty$. 
Suppose $1<p<\infty$. Define the operator family

$$
T_{z}:=|V|^{z p / 2}\left(H_{0}-\lambda\right)^{-1}|V|^{z p / 2},
$$

for $z \in \mathbb{C}$ with $0 \leq \Re z \leq 1$. Note that $T_{z}$ is continuous in the closed strip $0 \leq \Re z \leq 1$ and analytic in its interior. Moreover, estimating similarly as above, we get

$$
\sup _{0 \leq \Re z \leq 1}\left\|T_{z}\right\| \leq \frac{\max \left(1,\|v\|_{\infty}^{p}\right)}{\operatorname{dist}(\lambda,[-2,2])} .
$$

Therefore, $T_{z}$ is uniformly bounded for $0 \leq \Re z \leq 1$.

Further, since $v \in \ell^{p}(\mathbb{Z})$ by the hypothesis, we have $|v|^{p} \in \ell^{1}(\mathbb{Z})$. Consequently, we can apply 3.2 to get

$$
\left\|T_{1+\mathrm{i} y}\right\|=\left\||V|^{p / 2}\left(H_{0}-\lambda\right)^{-1}|V|^{p / 2}\right\| \leq \frac{\|v\|_{\ell^{p}(\mathbb{Z})}^{p}}{\sqrt{\left|\lambda^{2}-4\right|}},
$$

for any $y \in \mathbb{R}$. Moreover, for all $y \in \mathbb{R}$, we have also the trivial estimate

$$
\left\|T_{\mathrm{i} y}\right\| \leq \frac{1}{\operatorname{dist}(\lambda,[-2,2])} .
$$

Hence, we may apply Theorem 5 with $\theta=1 / p$ which yields

$$
\left\|T_{1 / p}\right\|=\|K(\lambda)\| \leq \frac{\|v\|_{\ell^{p}(\mathbb{Z})}}{\left|\lambda^{2}-4\right|^{\frac{1}{2 p}} \operatorname{dist}(\lambda,[-2,2])^{1-\frac{1}{p}}}
$$

and 2.4 completes the proof.

\section{Optimality of the eigenvalue bound for $\ell^{1}$-Potentials}

In this section, we demonstrate that the result (1.1) is sharp in the sense that to any boundary point of the spectral enclosure which is not located in $[-2,2]$, there exists an $\ell^{1}$-potential $V$ so that this boundary point is an eigenvalue of $H_{V}$. The excluded boundary points occur only if $\|v\|_{\ell^{1}(\mathbb{Z}) \mid} \leq 2$ and they are $\pm \sqrt{4-\|v\|_{\ell^{1}(\mathbb{Z})}^{2}}$.

For this purpose, we consider the operator $H_{V}$ with the potential $V$ determined by the sequence

$$
v_{n}:=\omega \delta_{n, 0}, \quad \forall n \in \mathbb{Z}
$$

where $\omega \in \mathbb{C}$ is a coupling constant and $\delta_{m, n}$ stands for the Kronecker delta. The eigenvalue equation $H_{V} \psi=\lambda \psi$ reads

$$
\psi_{n+1}+\omega \delta_{n, 0} \psi_{n}+\psi_{n-1}=\lambda \psi_{n}, \quad n \in \mathbb{Z} .
$$

It is convenient to write $\omega=k^{-1}-k$ for $0<|k| \leq 1$. Such $k$ exists unique inside the unit disk $|k|<1$ if $\omega \in \mathbb{C} \backslash[-2 \mathrm{i}, 2 \mathrm{i}]$, there are exactly two on the unit circle $|k|=1$, $k \neq \pm \mathrm{i}$ if $\omega \in(-2 \mathrm{i}, 2 \mathrm{i})$, and $k= \pm \mathrm{i}$ if $\omega=\mp 2 \mathrm{i}$. In any case, one readily verifies that the vector

fulfills the eigenvalue equation

$$
\psi_{n}:=k^{|n|}, \quad n \in \mathbb{Z}
$$

$$
H_{V} \psi=\left(k+k^{-1}\right) \psi .
$$

If, in addition, $\omega \in \mathbb{C} \backslash[-2 \mathrm{i}, 2 \mathrm{i}]$, then $\psi \in \ell^{2}(\mathbb{Z})$ and hence

$$
\lambda_{\omega}:=k+k^{-1}=\sqrt{4+\omega^{2}} \in \sigma_{\mathrm{p}}\left(H_{V}\right) .
$$

Obviously, $\left|\lambda_{\omega}^{2}-4\right|=|\omega|^{2}=\|q\|_{\ell^{1}(\mathbb{Z})}^{2}$ and thus the eigenvalue $\lambda_{\omega}$ is a boundary point of the spectral enclosure from (1.1). Moreover, let us remark that, in this particular example, the Birman-Schwinger operator takes the simple form

$$
K_{m, n}(\lambda)= \begin{cases}\frac{\omega}{k-k^{-1}} & \text { if } m=n=0 \\ 0 & \text { otherwise }\end{cases}
$$


for $\lambda=k+k^{-1}$ with $0<|k|<1$. Thus the Birman-Schwinger principle (2.3) together with the fact that $\sigma\left(H_{0}\right)=[-2,2]$ shows

$$
\sigma\left(H_{V}\right)=[-2,2] \cup\left\{\lambda_{\omega}\right\},
$$

where $\lambda_{\omega} \in[-2,2]$ if and only if $\omega \in[-2 \mathrm{i}, 2 \mathrm{i}]$, in which case $\lambda_{\omega} \notin \sigma_{\mathrm{p}}\left(H_{V}\right)$.

On the contrary, it is not difficult to see that to any boundary point $z \notin[-2,2]$ of the spectral enclosure $(1.1)$ for $H_{V}$ with $\|v\|_{\ell^{1}}>0$, there exists $k \in \mathbb{C}$ with $0<|k|<1$ and such that $z=k+k^{-1}$ and $\left|k-k^{-1}\right|=\|v\|_{\ell^{1}}$. Hence, if we put $\omega:=k^{-1}-k$, the previous analysis shows that $z$ is an eigenvalue of $H_{V}$ with the potential $V$ given by the sequence 4.1.

\section{Plots of the spectral enclosures from Theorems 1, 2, and 3}

First Figure 1 shows the spectral enclosures from Theorem 1 .

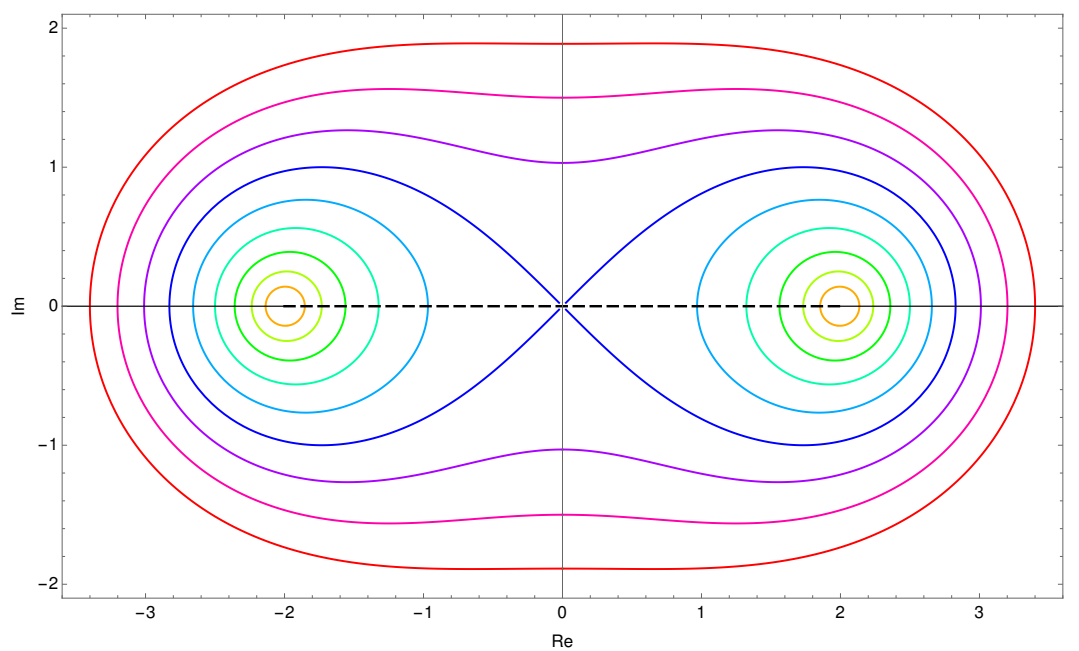

FIGURE 1. The plots of the expanding boundary curves corresponding to the spectral enclosure 1.1 for $\|v\|_{\ell^{1}(\mathbb{Z})}=j / 4, j=3, \ldots, 11$.

Second, we provide several plots as an illustration of the spectral enclosures from Theorem 2 in Figure 2. Denoting by $Q:=\|v\|_{\ell^{p}(\mathbb{Z})}$, the set in 1.2 is determined by two parameters $Q \geq 0$ and $q \geq 1$. Without going into details, we remark that the boundary curve $\Gamma_{q, Q}$ given by the equation

$$
\left|k-\frac{1}{k}\right|\left(\frac{1-|k|^{q}}{1+|k|^{q}}\right)^{1 / q}=Q
$$

for $0<|k|<1$, can be parametrized in the first quadrant $(\Re z \geq 0$ and $\Im z \geq 0)$ as

$$
\begin{aligned}
& \Re \Gamma_{q, Q}(t)=2 \cosh (t) \sqrt{\cosh ^{2}(t)-\frac{Q^{2}}{4} \operatorname{coth}^{\frac{2}{q}}\left(\frac{q t}{2}\right)}, \\
& \Im \Gamma_{q, Q}(t)=2 \sinh (t) \sqrt{-\sinh ^{2}(t)+\frac{Q^{2}}{4} \operatorname{coth}^{\frac{2}{q}}\left(\frac{q t}{2}\right)},
\end{aligned}
$$

for $t_{\min } \leq t \leq t_{\max }$, where $t_{\min }$ and $t_{\max }$ are the unique solutions of the equations

$$
\cosh ^{2}(t)=\frac{Q^{2}}{4} \operatorname{coth}^{\frac{2}{q}}\left(\frac{q t}{2}\right), \quad \text { and } \quad \sinh ^{2}(t)=\frac{Q^{2}}{4} \operatorname{coth}^{\frac{2}{q}}\left(\frac{q t}{2}\right),
$$

for $t>0$, respectively. This parametrization is used in the plots in Figure 2 . 
Third set of plots shows the spectral enclosures from Theorem 3 , see Figure 3 . Finally, we compare the spectral enclosures from Theorems 2 and 3 for six values of $p$ in Figure 4 The plots indicate that, for general $p>1$, no spectral enclosure is a subset of the other one.

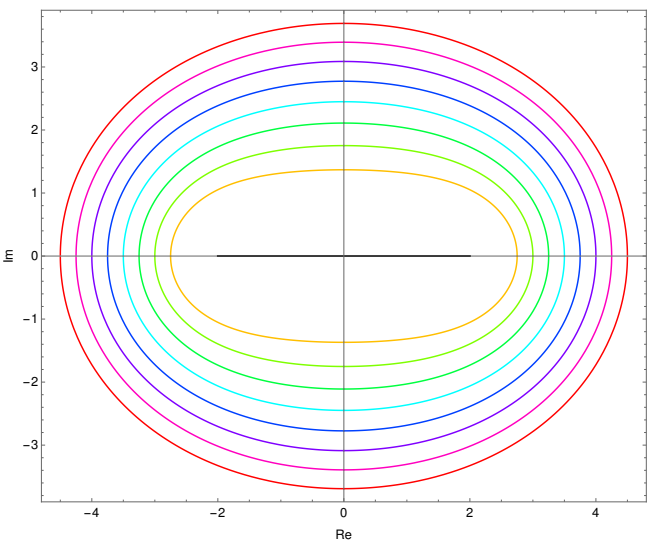

(A) $q=1$

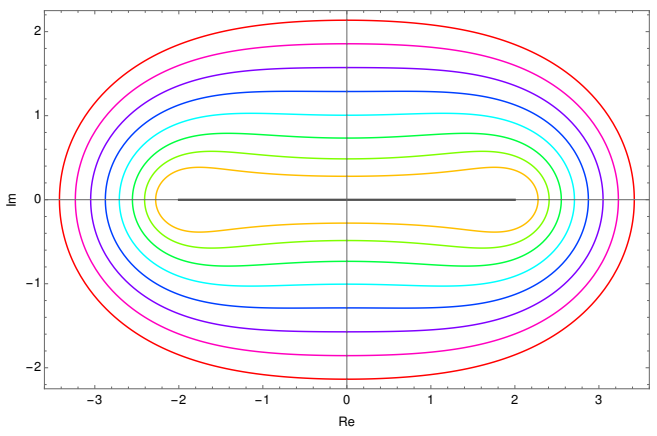

(c) $q=2$

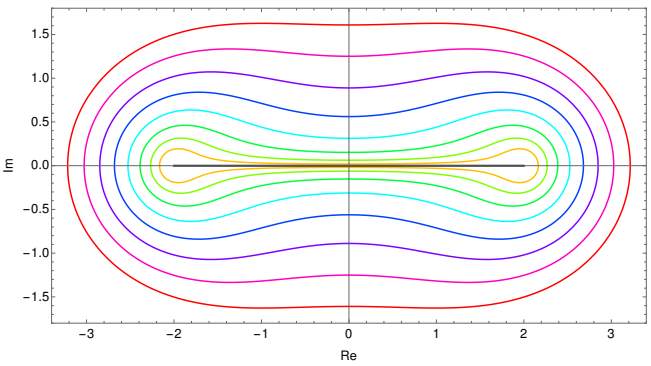

(E) $q=4$

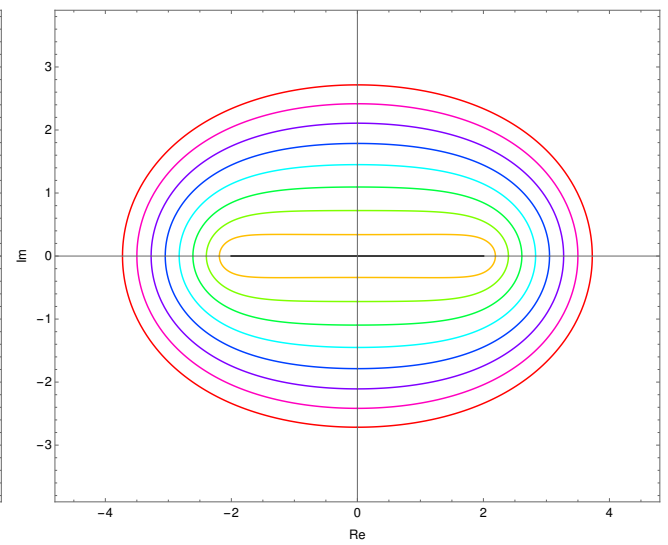

(в) $q=9 / 8$

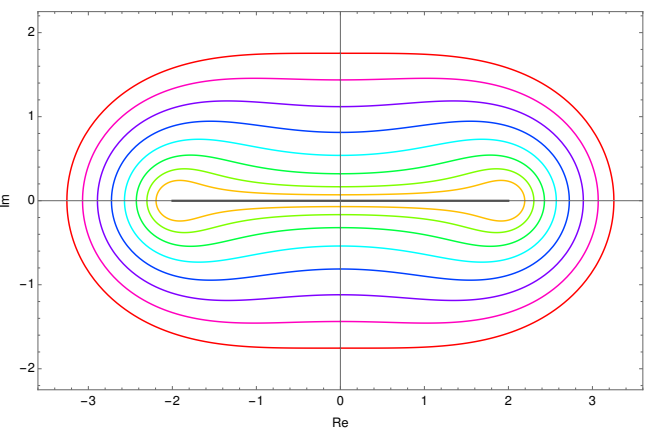

(D) $q=3$

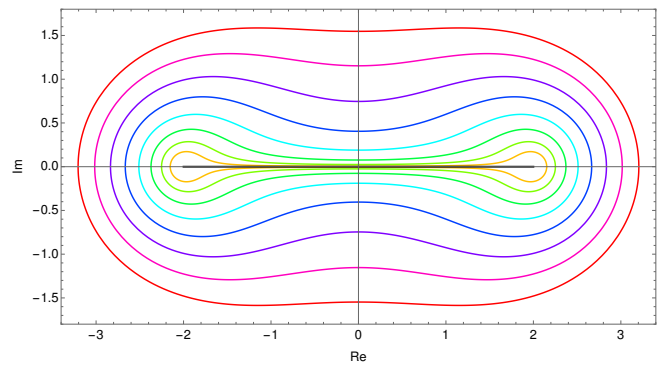

(F) $q=5$

FIGURE 2. The plots of the expanding boundary curves corresponding to the spectral enclosure $\sqrt{1.2}$ for six choices of the parameter $q$ and $\|v\|_{\ell^{p}(\mathbb{Z})}=j / 4$, $j=3, \ldots, 10$. 


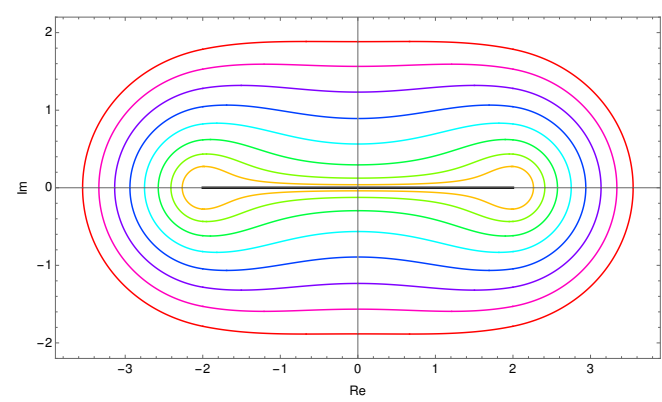

(A) $p=4 / 3$

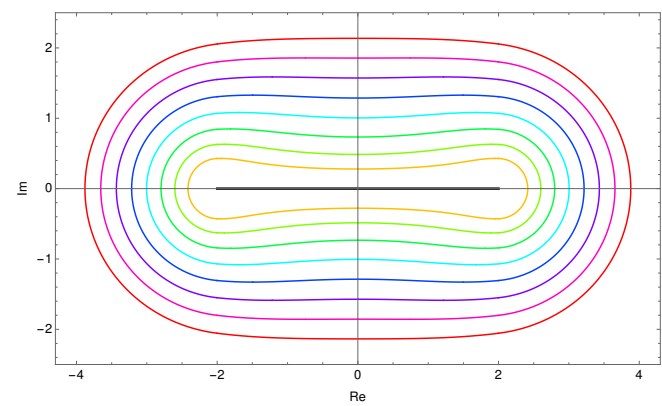

(C) $p=2$

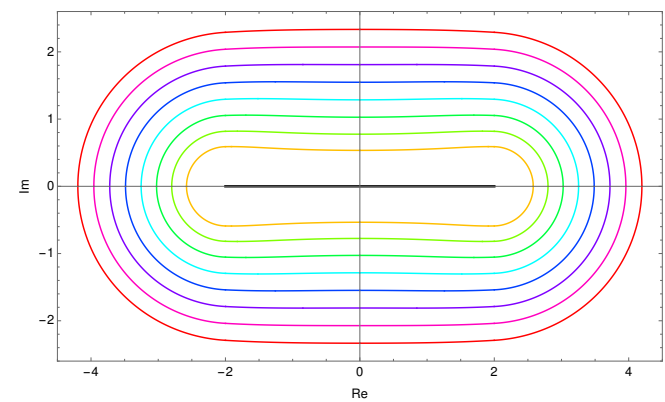

(E) $p=4$

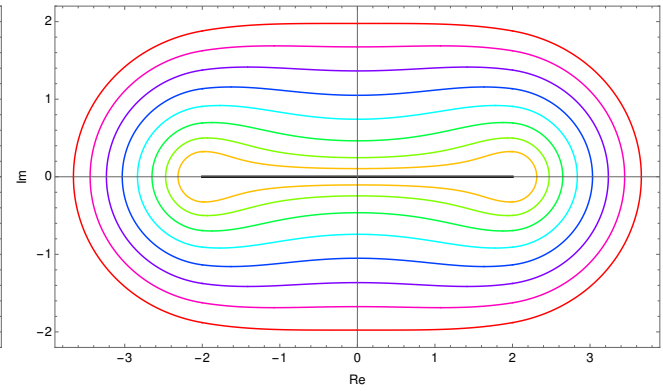

(B) $p=3 / 2$

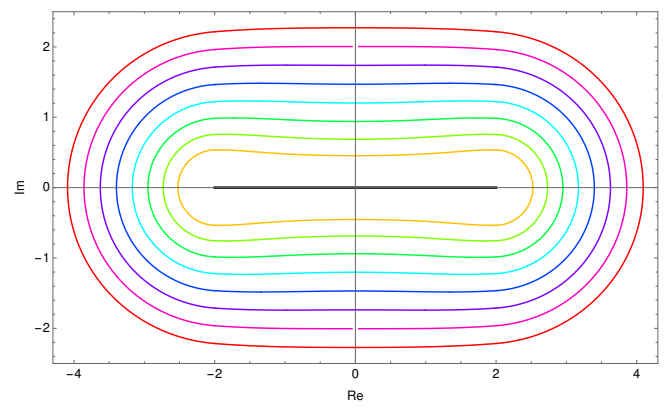

(D) $p=3$

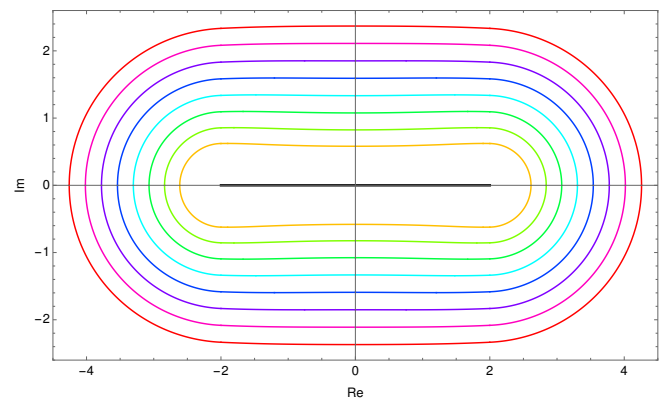

(F) $p=5$

FIGURE 3. The plots of the expanding boundary curves corresponding to the spectral enclosure 1.3 for six choices of the parameter $p$ and $\|v\|_{\ell^{p}(\mathbb{Z})}=j / 4$, $j=3, \ldots, 10$.

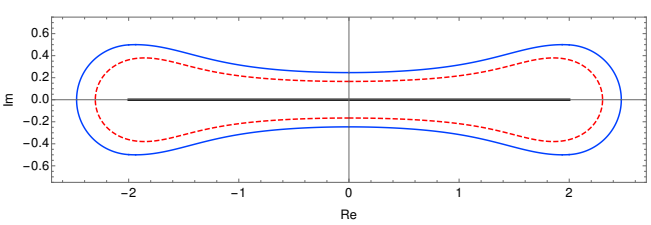

(A) $p=3 / 2$

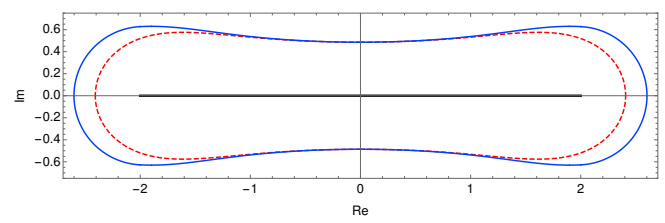

(B) $p=2$ 


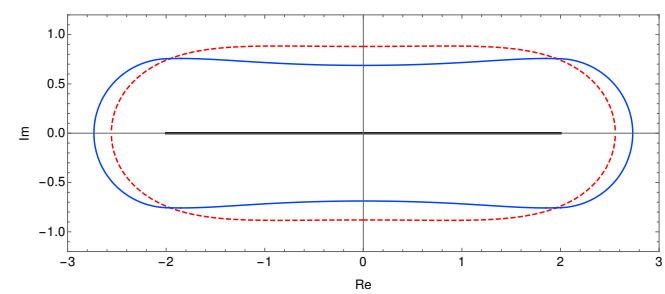

(C) $p=3$

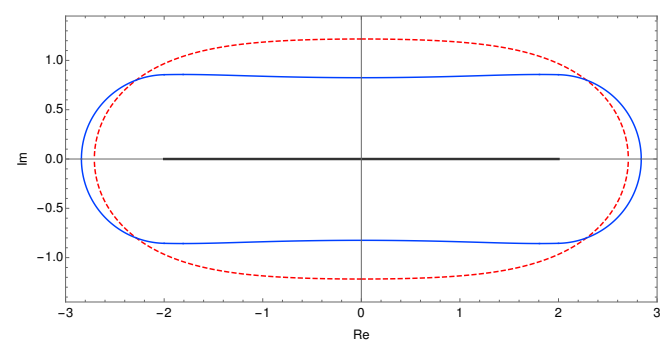

(E) $p=5$

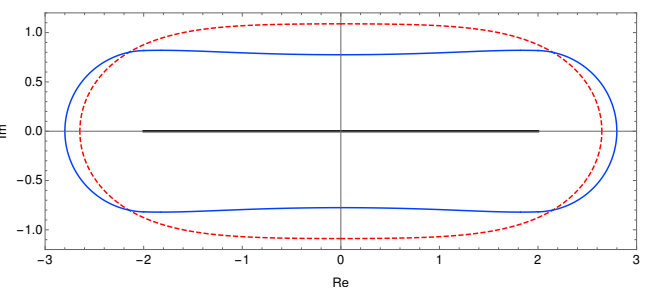

(D) $p=4$

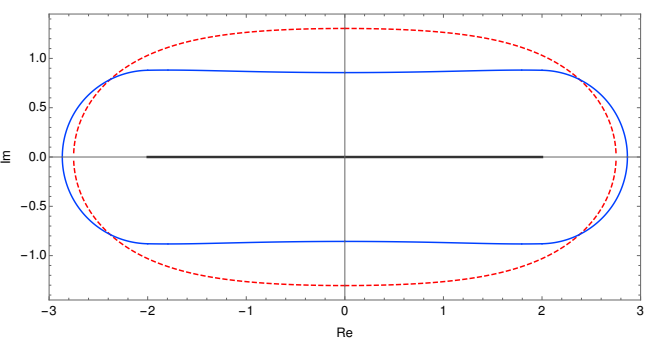

(F) $p=6$

FIGURE 4. A comparison of the boundary curves of the spectral enclosure 1.2 (red dashed line) with the spectral enclosure 1.3 (blue solid line) for six choices of the parameter $p$ and $\|v\|_{\ell^{p}(\mathbb{Z})}=1$.

\section{ACKNOWLEDGEMENT}

The first author thanks Prof. David Krejčiř́k for stimulating discussions. The second author acknowledges financial support by the Ministry of Education, Youth and Sports of the Czech Republic project no. CZ.02.1.01/0.0/0.0/16_019/0000778.

\section{REFERENCES}

1. A. A. Abramov, A. Aslanyan, and E. B. Davies, Bounds on complex eigenvalues and resonances, J. Phys. A 34 (2001), no. 1, 57-72.

2. J. Behrndt, M. Langer, V. Lotoreichik, and J. Rohleder, Spectral enclosures for non-self-adjoint extensions of symmetric operators, J. Funct. Anal. 275 (2018), no. 7, 1808-1888.

3. S. Bögli, Schrödinger operator with non-zero accumulation points of complex eigenvalues, Comm. Math. Phys. 352 (2017), no. 2, 629-639.

4. B. Cassano, O. O. Ibrogimov, D. Krejčiřík, and F. Štampach, Location of eigenvalues of non-selfadjoint discrete Dirac operators, arXiv:1910.10710 [math.SP] (2019).

5. J. B. Conway, A course in functional analysis, second ed., Graduate Texts in Mathematics, vol. 96 , Springer-Verlag, New York, 1990. MR 1070713

6. J.-C. Cuenin, Estimates on complex eigenvalues for Dirac operators on the half-line, Integr. Equ. Oper. Theory 79 (2014), no. 3, 377-388.

7. __ Eigenvalue bounds for Dirac and fractional Schrödinger operators with complex potentials, J. Funct. Anal. 272 (2017), no. 7, 2987-3018.

8. Sharp spectral estimates for the perturbed Landau Hamiltonian with $L^{p}$ potentials, Integr. Equ. Oper. Theory 88 (2017), no. 1, 127-141.

9. J.-C. Cuenin and C. E. Kenig, $L^{p}$ resolvent estimates for magnetic Schrödinger operators with unbounded background fields, Comm. Partial Differential Equations 42 (2017), no. 2, 235-260.

10. J.-C. Cuenin, A. Laptev, and C. Tretter, Eigenvalue estimates for non-selfadjoint Dirac operators on the real line, Ann. Henri Poincaré 15 (2014), no. 4, 707-736.

11. J.-C. Cuenin and C. Tretter, Non-symmetric perturbations of self-adjoint operators, J. Math. Anal. Appl. 441 (2016), no. 1, 235-258.

12. E. B. Davies and J. Nath, Schrödinger operators with slowly decaying potentials, J. Comput. Appl. Math. 148 (2002), no. 1, 1-28, On the occasion of the 65th birthday of Professor Michael Eastham.

13. M. Demuth, M. Hansmann, and G. Katriel, On the discrete spectrum of non-selfadjoint operators, J. Funct. Anal. 257 (2009), no. 9, 2742-2759. 
14. __ Eigenvalues of non-selfadjoint operators: a comparison of two approaches, Mathematical physics, spectral theory and stochastic analysis, Oper. Theory Adv. Appl., vol. 232, Birkhäuser/Springer Basel AG, Basel, 2013, pp. 107-163.

15. I. Egorova and L. Golinskii, On the location of the discrete spectrum for complex Jacobi matrices, Proc. Amer. Math. Soc. 133 (2005), no. 12, 3635-3641.

16. A. Enblom, Estimates for eigenvalues of Schrödinger operators with complex-valued potentials, Lett. Math. Phys. 106 (2016), no. 2, 197-220.

17. L. Fanelli and D. Krejčiř́k, Location of eigenvalues of three-dimensional non-self-adjoint Dirac operators, Lett. Math. Phys. 109 (2019), no. 7, 1473-1485.

18. L. Fanelli, D. Krejčiř́k, and L. Vega, Absence of eigenvalues of two-dimensional magnetic Schrödinger operators, J. Funct. Anal. 275 (2018), no. 9, 2453-2472.

19. L. Fanelli, D. Krejčiřík, and L. Vega, Spectral stability of Schrödinger operators with subordinated complex potentials, J. Spectr. Theory 8 (2018), no. 2, 575-604.

20. R. L. Frank, Eigenvalue bounds for Schrödinger operators with complex potentials, Bull. Lond. Math. Soc. 43 (2011), no. 4, 745-750.

21. E Eigenvalue bounds for Schrödinger operators with complex potentials. III, Trans. Amer. Math. Soc. 370 (2018), no. 1, 219-240.

22. R. L. Frank and R. Laptev, A.and Seiringer, A sharp bound on eigenvalues of Schrödinger operators on the half-line with complex-valued potentials, Spectral theory and analysis, Oper. Theory Adv. Appl., vol. 214, Birkhäuser/Springer Basel AG, Basel, 2011, pp. 39-44.

23. R. L. Frank and B. Simon, Eigenvalue bounds for Schrödinger operators with complex potentials. II, J. Spectr. Theory 7 (2017), no. 3, 633-658.

24. M. Hansmann, An eigenvalue estimate and its application to non-selfadjoint Jacobi and Schrödinger operators, Lett. Math. Phys. 98 (2011), no. 1, 79-95.

25. R. Henry and D. Krejčiřík, Pseudospectra of the Schrödinger operator with a discontinuous complex potential, J. Spectr. Theory 7 (2017), no. 3, 659-697.

26. A. Hulko, On the number of eigenvalues of the discrete one-dimensional Schrödinger operator with a complex potential, Bull. Math. Sci. 7 (2017), no. 2, 219-227.

27. O. O. Ibrogimov, D. Krejčiřík, and A. Laptev, Sharp bounds for eigenvalues of biharmonic operators with complex potentials in low dimensions, Preprint, ArXiv:1903.01810 (2019).

28. E. Korotyaev and A. Laptev, Trace formulae for Schrödinger operators with complex-valued potentials on cubic lattices, Bull. Math. Sci. 8 (2018), no. 3, 453-475.

29. D. Krejčiř́ík and P. Siegl, Pseudomodes for Schrödinger operators with complex potentials, J. Funct. Anal. 276 (2019), no. 9, 2856-2900.

30. A. Laptev and O. Safronov, Eigenvalue estimates for Schrödinger operators with complex potentials, Comm. Math. Phys. 292 (2009), no. 1, 29-54.

31. Y. Lee and I. Seo, A note on eigenvalue bounds for Schrödinger operators, J. Math. Anal. Appl. 470 (2019), no. 1, 340-347.

32. E. H. Lieb and M. Loss, Analysis, second ed., Graduate Studies in Mathematics, vol. 14, American Mathematical Society, Providence, RI, 2001.

33. O. Safronov, Estimates for eigenvalues of the Schrödinger operator with a complex potential, Bull. Lond. Math. Soc. 42 (2010), no. 3, 452-456.

34. B. Simon, Orthogonal polynomials on the unit circle. Part 2, American Mathematical Society Colloquium Publications, vol. 54, American Mathematical Society, Providence, RI, 2005, Spectral theory.

35. E. M. Stein, Interpolation of linear operators, Trans. Amer. Math. Soc. 83 (1956), $482-492$.

36. G. Teschl, Jacobi operators and completely integrable nonlinear lattices, Mathematical Surveys and Monographs, vol. 72, American Mathematical Society, Providence, RI, 2000.

(O. O. Ibrogimov) Department of Mathematics, Faculty of Nuclear Sciences and Physical Engineering, Czech Technical University in Prague, Trojanova 13, 12000 Praha 2, Czech REPUBLIC

E-mail address: ibrogori@fjfi.cvut.cz

(F. Štampach) Department of Applied Mathematics, Faculty of Information Technology,

Czech Technical University in Prague, Thákurova 9, 16000 Praha, Czech Republic

E-mail address: stampfra@fit.cvut.cz 